EPJ Web of Conferences 45, 01103 (2013)

DOI: $10.1051 /$ epjconf/20134501103

(C) Owned by the authors, published by EDP Sciences, 2013

\title{
Simulation of Flow around Isolated Helicopter Fuselage
}

\author{
A.N. Kusyumov ${ }^{1 a}$, S.A. Mikhailov ${ }^{1}$, E.V. Romanova ${ }^{1}$, A.O. Garipov ${ }^{2}$, E.I. Nikolaev ${ }^{2}$, G. Barakos ${ }^{3}$ \\ ${ }^{1}$ Kazan National Research Technical University n.a. A.N.Tupolev (KNRTU), Department of aero-hydrodynamics, Kazan, \\ Russia \\ 2 JSC Kazan Helicopters, Kazan, Russia \\ ${ }^{3}$ University of Liverpool, Department of Engineering, Liverpool, UK
}

\begin{abstract}
Low fuselage drag has always been a key target of helicopter manufacturers. Therefore, this paper focuses on CFD predictions of the drag of several components of a typical helicopter fuselage. In the first section of the paper, validation of the obtained CFD predictions is carried out using wind tunnel measurements. The measurements were carried out at the Kazan National Research Technical University n.a. A. Tupolev. The second section of the paper is devoted to the analysis of drag contributions of several components of the ANSAT helicopter prototype fuselage using the RANS approach. For this purpose, several configurations of fuselages are considered with different levels of complexity including exhausts and skids. Depending on the complexity of the considered configuration and CFD mesh both the multi-block structured HMB solver and the unstructured commercial tool Fluent are used. Finally, the effect of an actuator disk on the predicted drag is addressed.
\end{abstract}

\section{Introduction}

Following the 2020 goals of ACARE (Advisory Council for Aeronautics Research in Europe) the helicopter research community has renewed its interest in the aerodynamic analysis of helicopters [1]. Drag reduction is a primary goal and it is addressed using experimental and computational studies performed by a large number of researchers around Europe.

Experimental investigations of the helicopter fuselage aerodynamics usually provide information on integrated loads and some distributed parameters related to the flow structures around the body via velocity field explorations and spot measurements of the surface pressure. In contrast, Computational Fluid Dynamics (CFD) provides more detailed information of the three-dimensional flow. The ability to use CFD to predict drag and provide design engineers with accurate drag polars is therefore paramount.

A key element in helicopter performance is not only the overall drag, but also, the contributions to drag of individual fuselage components. Such analysis can lead to small re-designs with large potential performance benefits. In most cases, the isolated fuselage drag is treated in helicopter performance codes by scaling a D100 value (drag at 100 knots) to different conditions. This may be adequate at the initial helicopter design stages but it is limited if detailed predictions are necessary.

Further improvements could also come from estimating the effect of the main rotor wake on fuselage drag using a simple method that avoids detailed modeling the complex aerodynamics of the rotor blades. For this purpose the use of a simple momentum source method is used. This is not a new technique and has been used by several researchers in the rotor field [2-4].

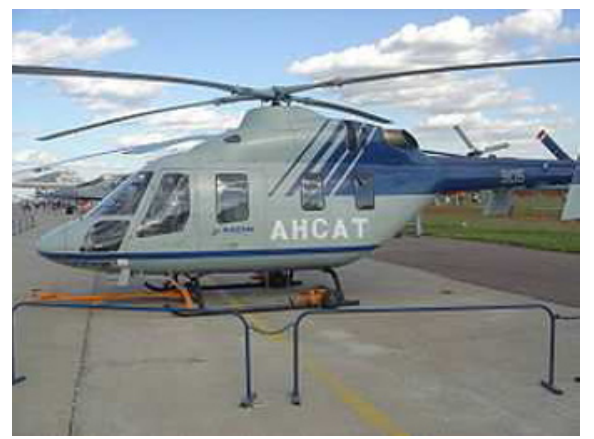

Fig. 1. Photo of ANSAT helicopter

As an example of CFD application to the analysis of helicopter fuselage drag, the flow around a representative fuselage is considered in this paper. Computations were performed using in-house as well as commercial tools. In order to have a fuselage representative of modern designs, an approximation ANSAT-P to the ANSAT helicopter produced by the JSC Kazan Helicopters (figure 1) is considered in this paper. Variations of this baseline fuselage the fuselage referred to as ANSAT-M (figure 2) are also used. ANSAT-M is one of the many initial designs used during the development of the ANSAT aircraft and was used for preliminary aerodynamic studies conducted in the wind tunnel T-1K of KNRTU. The fuselage is not the exact shape of the ANSAT helicopter body but it is a good approximation with main differences at the engine housing and exhausts.

\footnotetext{
1a postbox7@mail.ru
} 


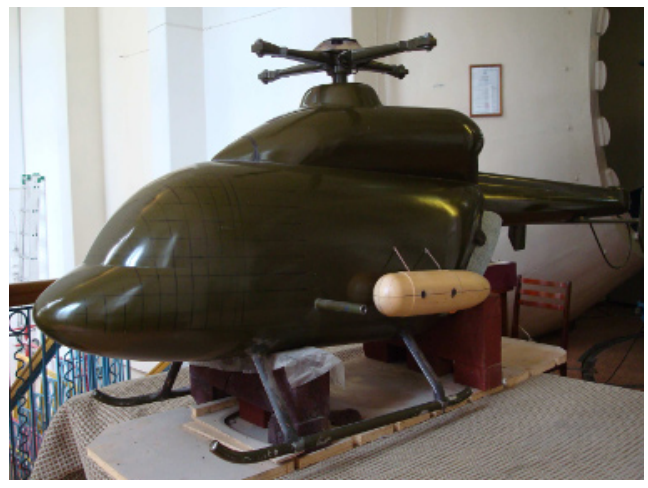

Fig. 2. Photo of ANSAT-M fuselage

The CFD grids were constructed using the ANSYS ICEM software. The entire domains were resolved using a tetra and hexa-grids and the 3D steady incompressible Reynolds-Averaged Navier-Stokes (RANS) equations. The computation of the drag generated by an isolated fuselage was the first step of the present investigation. Fully turbulent calculations were performed using the SpalartAllmaras (S-A), k- $\omega$ SST (SST) and transitional k-kl- $\omega$ (TR) models.

\section{Computations for isolated ANSAT-M fuselage}

The ANSAT-M model fuselage employed in this work was laser-scanned to provide the actual CAD model. The length of fuselage was $L_{F}=1.57 \mathrm{~m}$, a value $S_{F}=0.0694 \mathrm{~m}^{2}$ was used as reference area.

Figure 3 presents the idealized ANSAT-M fuselage without the horizontal stabilizer, undercarriage, fin, main and tail-rotor hubs. This is a substantial simplification of the overall problem complexity. On the other hand, it allows for easier mesh generation and economies in the employed CFD grid. The fuselage is also divided in several parts and the forces on each of these components can be analyzed separately. The parts are shown in different colours on figure 3 .

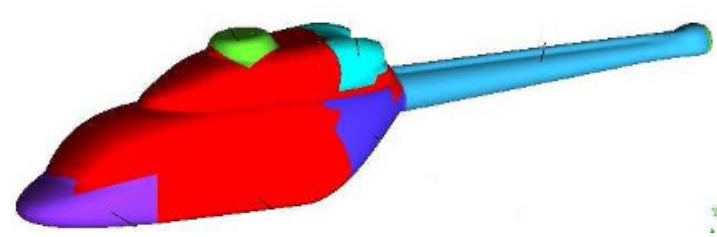

Fig. 3. CAD model of ANSAT-M fuselage

For the full model of the ANSAT-M fuselage, ICEMhexa produced grids with 764 blocks. For a grid sensitivity study, two grids were considered with: $6,450,000$ hexahedral cells $(216,500$ surface elements) and 9,200,000 hexahedral cells $(318,000$ surface elements). The value of $\mathrm{y}^{+}$for all employed turbulence models was up to 0.5 and this is considered adequate for resolving the laminar sublayer for better predictions of the friction drag component.
The baseline surface mesh of $6,450,000$ cells is presented in the figure 4 . The grid sensitivity study and validation of the employed modeling were performed at zero degrees angle of attack, and Reynolds number of $\operatorname{Re}=3,871,000$ (corresponding to a free stream velocity of $V_{\infty}=36 \mathrm{~m} / \mathrm{s}$ ).

Table 1 shows the predictions for the total drag and lift forces with respect to the number of surface points and in comparison with the wind tunnel experiment data [5]. Numerical modeling was conducted for transient formulation due to presence of strong separation of flow at the rare part of fuselage and the Table 1 presents some average values.

Table 1. Force coefficients for different cells numbers and turbulence models.

\begin{tabular}{|l|c|c|}
\hline & $\boldsymbol{C}_{\boldsymbol{D}}$ & $\boldsymbol{C}_{\boldsymbol{L}}$ \\
\hline 6,450,000 sells & & \\
S-A & 0.135 & -0.044 \\
SST & 0.162 & -0.062 \\
TR & 0.11 & -0.05 \\
\hline 9,200,000 cells & & \\
S-A & 0.15 & -0.026 \\
SST & 0.158 & -0.045 \\
TR & 0.135 & -0.035 \\
\hline WT experiment & 0.16 & -0.06 \\
\hline
\end{tabular}

The results show a moderate grid sensitivity for the SST models, while the rest of the models are more sensitive to the mesh refinement. In addition, the SST model predicted the values of aerodynamic coefficients closer to the experimental value in comparison with the SA and TR.

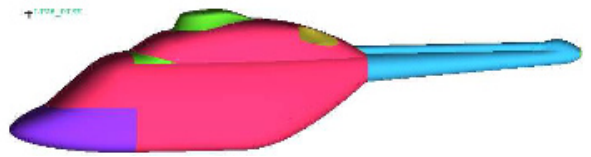

a)

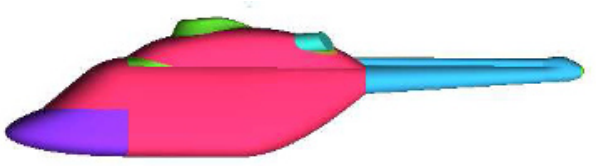

b)

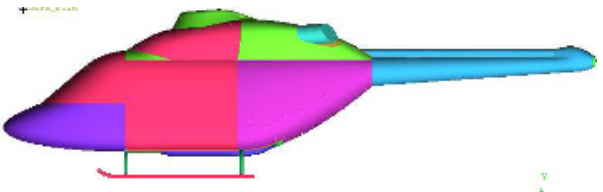

c)

Fig. 4. ANSAT-P fuselage models:

a) Configuration 1 - isolated fuselage without exhausts;

b) Configuration 2 - isolated fuselage with exhausts and actuator disk; c) Configuration 3 - isolated fuselage with exhausts, skid and fairing of fuel system 


\section{Computations for isolated ANSAT-P fuselage}

Figure 4 shows a close view of the ANSAT-P fuselage configurations, computed in this work. To better approximate the realistic ANSAT-P fuselage, the inlet and outlet of the engine compartment have been closed, and exhausts were added. A different version also included skids. The numerical computations are presently carried out without the rotor head.

For this case there were no experiments to compare against and for this reason, the baseline solutions obtained using the Fluent code and a tetra-mesh, were compared against the the HMB code of Liverpool University that employed a multi-block hexa-mesh. The grids have been generated by means of the commercial software ANSYS ICEM.

The tetra-grid contained 20 prism layers around the solid surfaces, necessary to model the boundary layer flow correctly. Figure 5 presents several views of the employed mesh for the most complex configuration.

The number of grid cells for the different configurations of fuselage layouts varies from about 3,015,000 cells for Configuration 1 , to $3,320,000$ for Configuration 2 and 4,311,000 for Configuration 3.

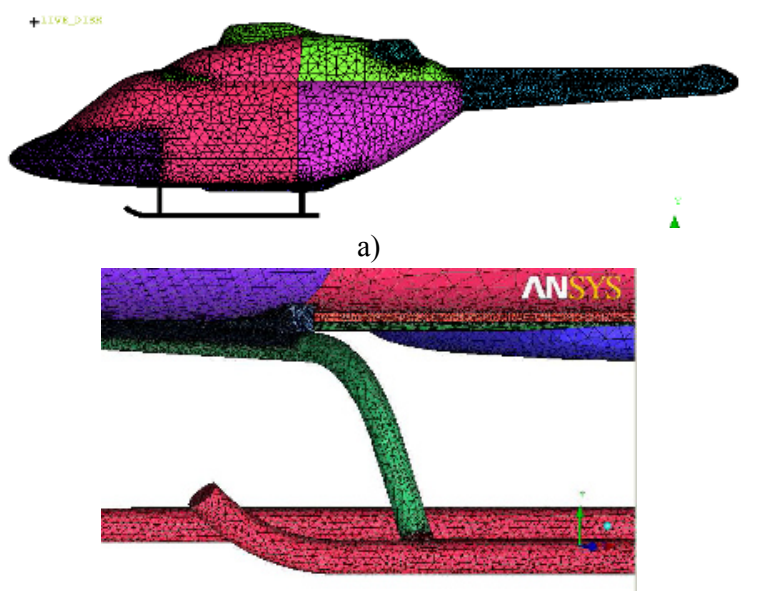

b)

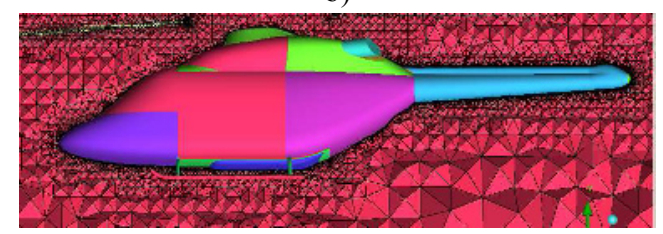

c)

Fig. 5. Mesh fragments for Configuration 3 fuselage: a) Fuselage surface mesh; b) Skid surface mesh;

c) Cut through volume mesh at position $z=0$

The value of $\mathrm{y}^{+}$for SST turbulence model was up to 0.7 . At least 15 elements were kept in the boundary layer for sufficient resolution. The mesh near the wall for this application of the $L_{F}=1.1 \mathrm{~m}$ fuselage length (the reference area is $S_{F}=0.04$ ) had the first element height of $0.005 \mathrm{~mm}$.
The hexa-grid was constructed for the half of Configuration 1 fuselage. For the half ANSAT-P fuselage a hexahedral mesh of 301 blocks has been used for meshing. The grid contains about 3,000,000 hexahedral cells (about 130,000 surface elements). The value of $\mathrm{y}^{+}$for $\mathrm{k}$-omega turbulence model was up to 0.5 to resolve the laminar sublayer for better predictions of the friction drag component. The mesh on the fuselage surface is presented in the figure 6 .

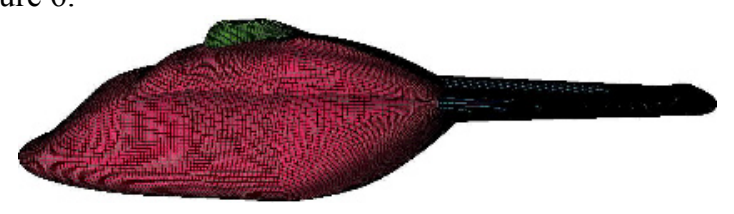

Fig. 6. Surface mesh for Configuration 1 fuselage

Computations were performed in a steady state incompressible formulation (for certain angles of attack solution has oscillating character, but deviations of the drag coefficients are not so significant). Figure 7 presents the results of the drag coefficient prediction versus of angle $\alpha$ of fuselage attack at two Reynolds numbers.

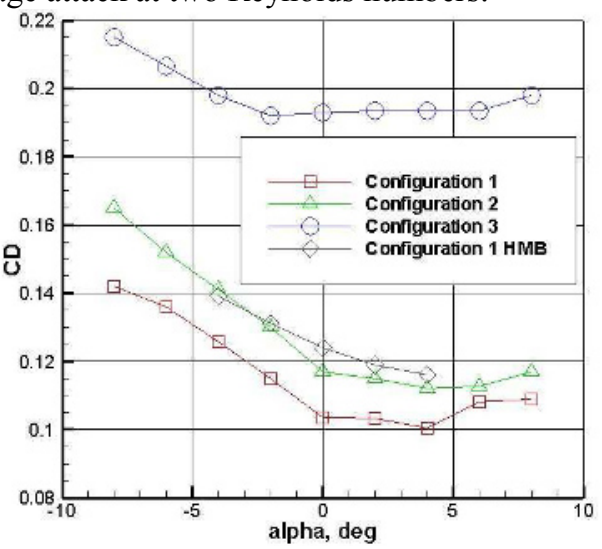

a)

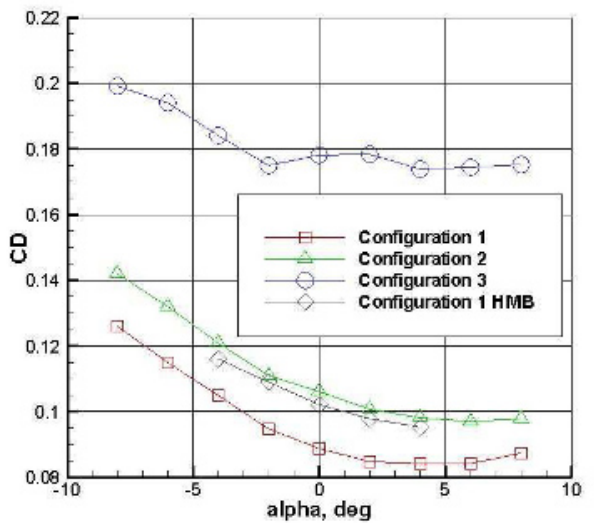

b)

Fig. 7. Drag coefficients predicted by Fluent and HMB codes: a) $\operatorname{Re}=1,055,000\left(V_{\infty}=14 \mathrm{~m} / \mathrm{s}\right)$, Fluent; $\mathrm{Re}=1,200,000$

$$
\left(M_{\infty}=0.1\right), \mathrm{HMB}
$$


b) $\operatorname{Re}=3,993,000\left(V_{\infty}=53 \mathrm{~m} / \mathrm{s}\right)$, Fluent; $\operatorname{Re}=4.700 .000$

$$
\left(M_{\infty}=0.1\right), \mathrm{HMB}
$$

Figure 7 presents also the results of the drag coefficient versus of angle $\alpha$ of fuselage attack prediction conducted in HMB code for Configuration 1 using a hexa-grid. Computations were performed for compressible formulation, free stream Mach number of $M_{\infty}=0.1$.

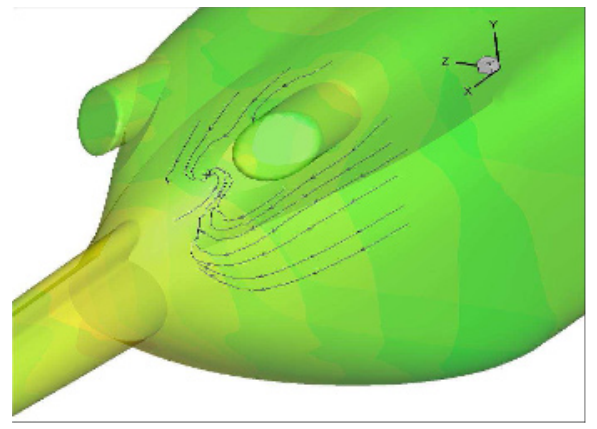

Fig. 8. Surface streamlines in the area of exhaust pipe

From the comparison of computations results for Configuration 1 it follows qualitative agreement between results predicted by Fluent and HMB codes. However the values of the drag coefficient have some discrepancy: the values for $C_{D}$ obtained in HMB are up to $10 \%$ higher than the values predicted by Fluent. The reason of discrepancy can be explained by different reasons: different formulation of the flow properties (incompressible flow for Fluent and compressible for HMB) and the quality of grids used for computations. A preliminary study using Fluent in compressible mode, showed a similar increase in the drag predictions by almost $10 \%$.

\section{Actuator disk computations}

The actuator disk approximates the rotor using an infinitely thin source of momentum. This is modeled here as a steady, pressure jump across the disk. At the moment, the momentum source was modeled independently of the fuselage without any feedback between the two. For this reason, the effect of the rotor disk is simplified and the method, although computationally efficient, can only be used for initial estimates of the fuselage loads [2, 3]. The well-know upwash-downwash mechanism highlighted in [6] is therefore not take into account here. To obtain the pressure jump for the mean rotor thrust the uniform actuator disk was modeled using basic momentum theory. The constant pressure jump was applied to the grid points of the actuator disk, inside a radius $R$ of $0.574 \mathrm{~m}$ (figure 9), with a disk tilt of 4.5 degrees. Computations were performed for two values of free stream velocity of 14 and $53 \mathrm{~m} / \mathrm{s}$ (on the 3.320 .000 cell grid). For these conditions the $C_{T} / \mu^{2}$ were 1.25 and $0.0872(\Delta p=300 \mathrm{~Pa})$, respectively. Here $C_{T}=T /\left(\rho \pi R^{2} V_{t}^{2}\right)$ is the trust coefficient, $\mu=V_{\infty} / V_{t}$ is the advanced ratio, $V_{t}$ is the rotor tip velocity, $\rho$ is the air density.

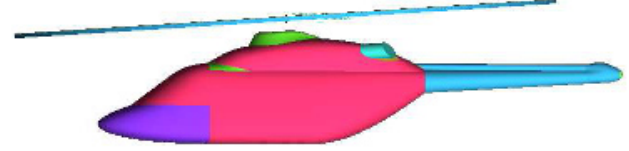

Fig. 9. Configuration 2 with the actuator disk

For a non-uniform disk the "typical" distribution of the pressure was estimated based on [7, 8]. The distribution of pressure jump along the disk surface is determined by the expression

$$
\Delta p=\rho \gamma\left[\gamma \operatorname{sign}(\delta) / 2+V_{\infty} \cos \left(\alpha-\alpha_{a}+\delta\right)\right] .
$$

Here $\delta$ is the angle of vortex cylinder slope $\alpha_{a}$ is the angle of actuator incidence. The function $\gamma$ is distribution of circulation on the disk surface:

$$
\gamma=\gamma_{r}+\gamma_{S} \sin (\psi)
$$

where $\psi$ is azimuth angle of considered point on disk surface.

The distribution of averaged blade load $\gamma_{r}$ can be written in the form [8]

$$
\gamma_{r}=C f_{r}\left(r_{n}\right)
$$

where $f_{r}\left(r_{n}\right)=r_{n}^{2}\left(2-r_{n}^{2}-r_{n}^{4}\right), r_{n}=r / R$ is normalized radius. The value of $C$ is determined by the formula

$$
C=1.989 V_{\infty}\left[-\cos \left(\alpha-\alpha_{a}+\delta\right)+\sqrt{\cos ^{2}\left(\alpha-\alpha_{a}+\delta\right)+1.27 C_{T} / \mu^{2}}\right] .
$$

The expression for $\gamma_{s}$ has the form

$$
\gamma_{s}=C B \mu_{v} f_{s}\left(r_{n}\right) \text {, }
$$

where

$$
\begin{aligned}
& B=\frac{8 \mu_{v}\left(1+k_{\delta}^{2}\right)+a_{\infty} \sigma k_{\delta}}{\left(1+k_{\delta}^{2}\right)\left(4 \mu_{v}+a_{\infty} \sigma k_{\delta}\right)}, k_{\delta}=\tan \left(\frac{\pi}{4}-\frac{|\delta|}{2}\right), \\
& f_{s}\left(r_{n}\right)=f_{r}\left(r_{n}\right)\left(r_{n}^{-1}-\frac{25}{13} r_{n}\right), \mu_{v}=\mu+\frac{v_{x a}}{\omega R} .
\end{aligned}
$$

The expression for $B$ coefficient contains the value $a_{\infty}$ of the lift coefficient slope. The average value of induced velocity $v_{x a}$ is estimated using

$$
v_{x a}=\frac{1}{2} \operatorname{sign}(\delta) v_{1 y a} \tan \left(\frac{\pi}{2}-\frac{|\delta|}{4}\right),
$$

where

$$
\begin{aligned}
& v_{1 y a}=\frac{1}{2} V_{\infty} \operatorname{sign}(\delta)\left[-\cos \left(\alpha-\alpha_{a}+\delta\right)+\right. \\
& \left.+\sqrt{\cos ^{2}\left(\alpha-\alpha_{a}+\delta\right)+\operatorname{sign}(\delta) C_{T} / \mu^{2}}\right] .
\end{aligned}
$$

Eventually in this paper it is assumed that the pressure distribution depends on the local disk radius $r$ only:

$$
\Delta p_{0}=\rho\left[\gamma_{r}^{2} / 2+\gamma_{s}^{2} / 4+\gamma_{r} V_{\infty} \cos \left(\alpha-\alpha_{a}+\delta\right)\right] .
$$


The non-uniform disk was implemented in the HMB solver and in Fluent using user-defined functions. The pressure coefficient $C_{p}=2\left(p-p_{\infty}\right) /\left(\rho V_{\infty}^{2}\right)$ distribution on the fuselage surface ( $p$ is a local pressure, $p_{\infty}$ is the free stream pressure) and the flow field are shown in figures 9-12.

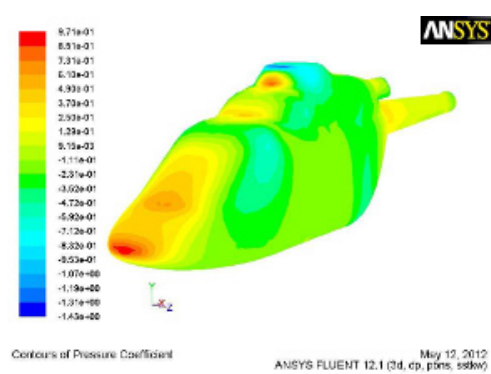

a)

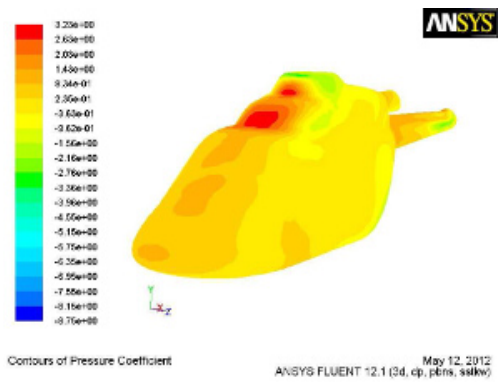

b)

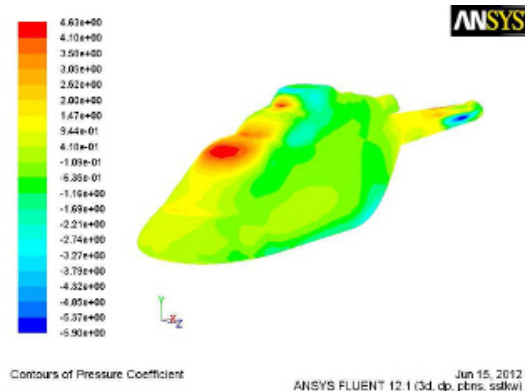

c)

Fig. 9. Pressure coefficient distribution on the fuselage surface for $\alpha=0$ degrees, $V_{\infty}=14 \mathrm{~m} / \mathrm{s}$ :

a) without the actuator disk; b) with the uniform actuator disk; c) with the non-uniform disk

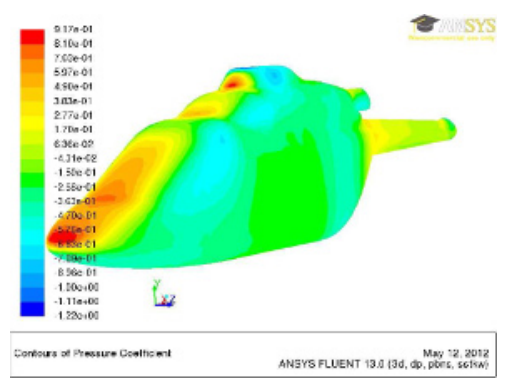

a)

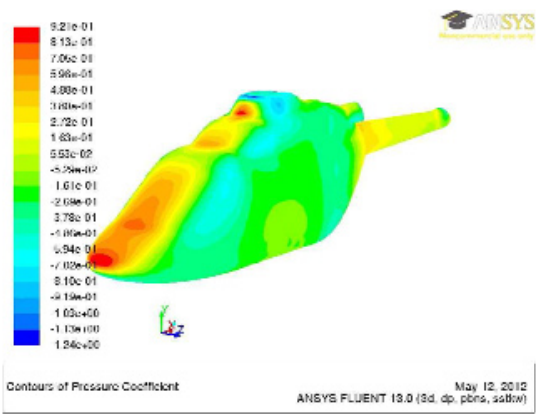

b)

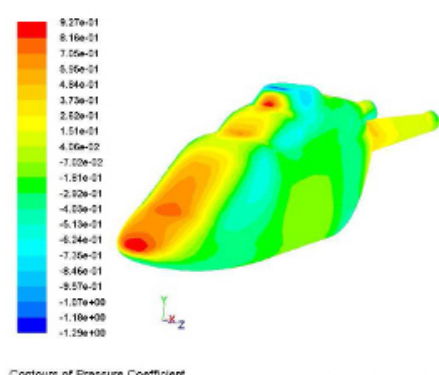

MNSYS

c)

Fig. 10. Pressure coefficient distribution on the fuselage surface for $\alpha=-8$ degrees, $V_{\infty}=53 \mathrm{~m} / \mathrm{s}$ :

a) without the actuator disk; b) with the uniform actuator disk; c) with the non-uniform disk
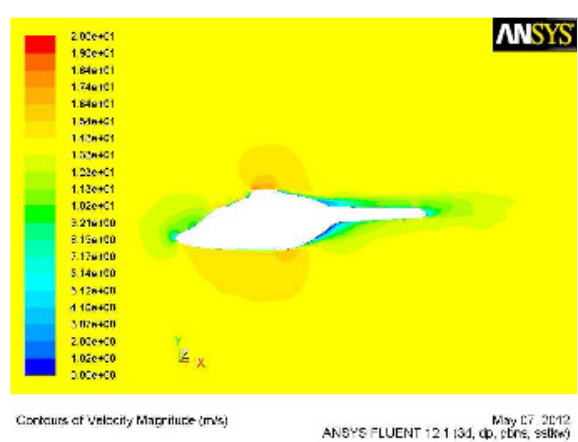

a)

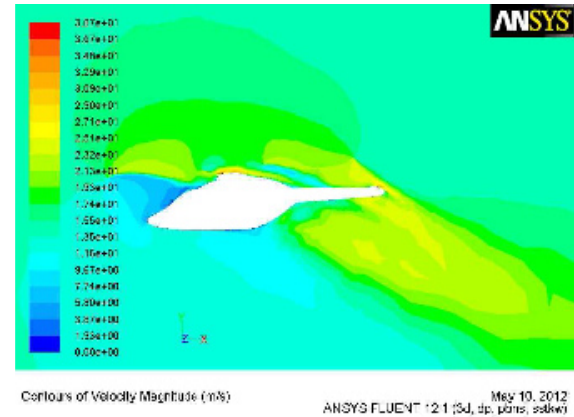

b) 


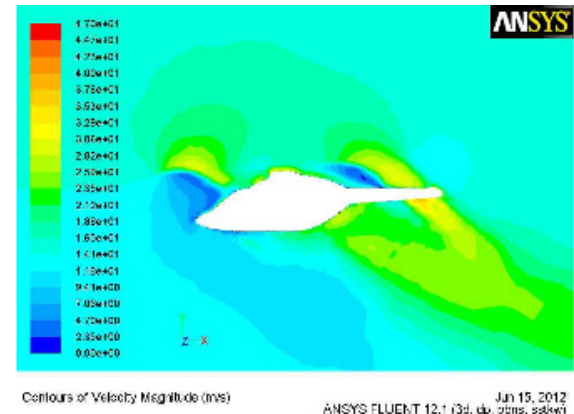

c)

Fig. 11. Velocity magnitude on the symmetry plane for the fuselage for $\alpha=0$ degrees, $V_{\infty}=14 \mathrm{~m} / \mathrm{s}$ :

a) without the actuator disk; b) with the uniform actuator disk; c) with the non-uniform disk

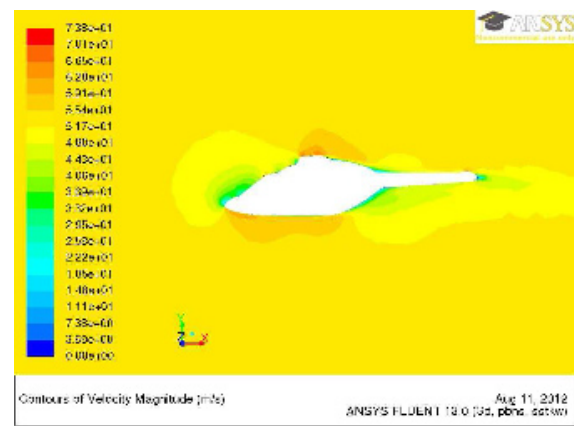

a)

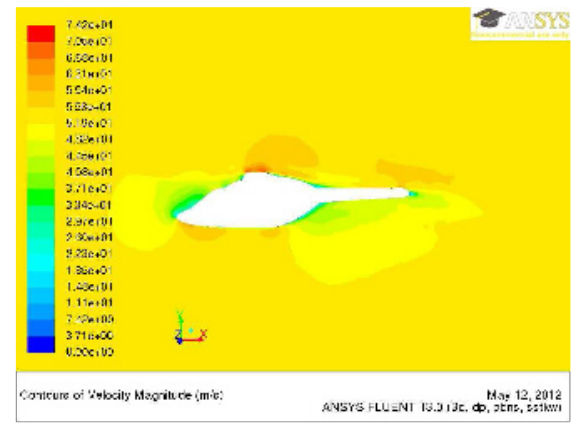

b)

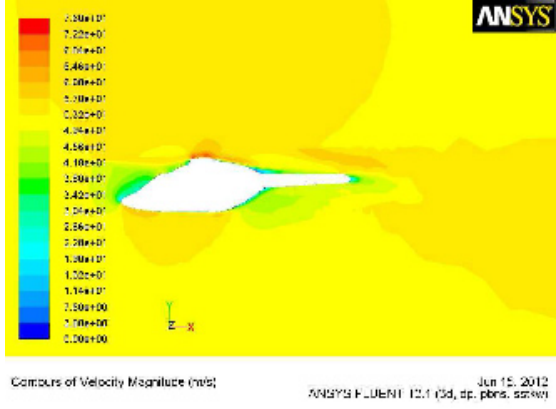

c)

Fig. 12. Velocity magnitude on the symmetry plane for the fuselage for $\alpha=-8$ degrees, $V_{\infty}=53 \mathrm{~m} / \mathrm{s}$ :

a) without the actuator disk; b) with the uniform actuator disk; c) with the non-uniform disk
Without the actuator, a decrease of stagnation pressure takes place behind the rear fuselage, and behind the tail, due to flow separation. This modifies the pressure distribution on the fuselage surface. The main differences between the isolated fuselage calculation and the calculation with the actuator disk can be seen on the upper part of the fuselage as a consequence of the rotor downwash. The rotor creates also a deflection of the flow downwards. The influence of the rotor downwash on the fuselage global forces and moments is also significant, especially on the drag and on the pitching moment, as discussed in $[9,10]$.

The predicted values of the fuselage drag coefficient with and without the actuator disk are presented in the Table 2 .

Table 2. Fuselage drag coefficient without and without the actuator disk

\begin{tabular}{|c|c|c|}
\hline$C_{D}$ & $\begin{array}{c}V_{\infty}=14 \mathrm{~m} / \mathrm{s}, \\
\alpha=0\end{array}$ & $\begin{array}{c}V_{\infty}=53 \mathrm{~m} / \mathrm{s}, \\
\alpha=-8\end{array}$ \\
\hline without & 0.119 & 0.146 \\
\hline uniform & 0.592 & 0.154 \\
\hline nonuniform & 0.813 & 0.153 \\
\hline
\end{tabular}

\section{Conclusions and future work}

The flow around two idealized fuselages of the ANSAT helicopter was analyzed for the fuselages of the ANSAT-M (early prototype) and a more realistic ANSAT-P model of three configurations. The fuselage drag was computed using different turbulence models and their results suggested substantial sensitivity.

From the comparison of the drag of the two fuselages it follows that the drag of the ANSAT-P fuselage is less than for the ANSAT-M. This reduction is due to the more streamlined shape of this body. For the ANSAT-P fuselage the problem was re-visited using a uniform and nonuniform actuator disk that changed the pressure distribution near stagnation points and consequently the relative importance of the fuselage parts. Although this was a crude modeling attempt, it serves to highlight the importance of having the correct inflow when the fuselage shape is investigated for the purposes of drag reduction.

In the future, the problem of the helicopter drag will be revisited with a better near-fuselage grid following the multi-block structured approach. This will allow for more accurate drag estimates as well as better estimates of the points of separation. The actuator disk model is also to be refined and update. Unsteady CFD is also to be undertaken to allow for resolving the separated flow region and revealing the periodic forcing on the fuselage.

\section{Acknowledgments}

This work is supported by the Grant according the Decree No. 220 of Russian Federation Government about Attracting Leading Scientists to Russian Educational Institutions (agreement No 11.G34.31.0038). 


\section{References}

1. S. Schneider, S. Mores, M. Edelmann, A. D'Alascio, D. Schimke, 37th European Rotorcraft Forum, (MAGA Galarate, Italy, 2011)

2. $\mathrm{Ph}$. Beaumier, J.-M. Bousquet, ICAS conference (2004)

3. T. Renaud, D. O'Brien, M. Smith, M. Potsdam, American Helicopter Society 60th Annual Forum (Baltimore, MD, 2004)

4. T. Renaud, C. Benoit, J.-C. Boniface and P. Gardarein, 29th European Rotorcraft Forum (Friedrichshaffen, Germany, 2003)

5. V. Pahov, M. Valiev, V. Zherekhov, L. Makarova, A. Kusuymov and G. Barakos, 47th Applied Aerodynamics Symposium, (Paris, France, 2012)

6. R. Steijl, G. Barakos, AIAA J. 47, 2143-2157 (2009)

7. H. Heyson, S. Katzoff, NACA TR, 1319 (1957)

8. V.I. Shaidakov, Proceedings of Moscow Aviation Institute, 31, (1978) [in Russian]

9. C.E. Freeman, R.E. Mineck, NASA Technical Memorandum, 80051 (1979)

10. R. Steijl, G. Barakos, Int. J. Numer. Meth. Fluids, 58, 527-549 (2008) 